\title{
Hydrodynamic signal perception in the copepod Acartia tonsa
}

\author{
Thomas Kiørboe ${ }^{1, *}$, Enric Saiz ${ }^{2}$, André Visser ${ }^{1}$ \\ ${ }^{1}$ Danish Institute For Fisheries Research, Charlottenlund Castle, DK-2920 Charlottenlund, Denmark \\ ${ }^{2}$ Institut de Ciències del Mar, CSIC, Passeig Joan de Borbó s/n, E-08039 Barcelona, Spain
}

\begin{abstract}
Copepods may remotely detect predators from the velocity gradients these generate in the ambient water. Each of the different components and characteristics of a velocity gradient (acceleration, vorticity, longitudinal and shear deformation) can cause a velocity difference between the copepod and the ambient water and may, therefore, be perceived by mechanoreceptory setae. We hypothesised that the threshold value for escape response to a particular component depends solely on the magnitude of the velocity difference (= signal strength) it generates. In experiments we isolated the different components and noted the minimum intensities to which the copepod Acartia tonsa responded. As hypothesised, threshold signal strengths due to longitudinal and shear deformation were similar, $\sim 0.015 \mathrm{~cm} \mathrm{~s}^{-1}$, and were invariant with developmental stage. The latter implies that the threshold deformation rate for response scales inversely with size, i.e. that large stages respond to lower fluid deformation rates than small stages and, hence, may detect predators at longer distances. Signals due to vorticity and acceleration did not elicit escape responses, even though their magnitude exceeded threshold signal strength due to deformation. We suggest that $A$. tonsa cannot distinguish such signals from those due to their own behaviour (sinking, swimming, passive reorientation due to gravity) because they cause a similar spatial distributions of the signal across the body. Reinterpretation of data from the literature revealed that threshold signal strength due to deformation varies by ca 2 orders of magnitude between copepods and exceeds the neurophysiological response threshold by more than a factor of 10 . In contrast, threshold deformation rates vary much less, $\sim 0.5$ to $5 \mathrm{~s}^{-1}$ Model calculations suggest that such threshold deformation rates are just sufficient to allow efficient predator detection while at the same time just below maximum turbulent deformation rates, thus preventing inordinate escapes
\end{abstract}

KEY WORDS: Predator detection - Threshold deformation rate - Turbulence

\section{INTRODUCTION}

Copepods may remotely detect an approaching predator by the hydromechanical disturbances it generates in the fluid (Kerfoot 1978, Haury et al. 1980). The magnitude of the disturbance required for perception and to elicit an escape response determines the distance at which the copepod can react to the predator. Obviously, this distance may be crucial to the chance of the copepod escaping the predator.

A moving predator generates velocity gradients in the ambient fluid. Such velocity gradients may be perceived by a copepod because gradients in velocity

•E-mail: tk@dfu.min.dk cause a velocity difference between the copepod and the ambient water (Kiørboe \& Visser 1999 in this issue). This velocity difference, in turn, causes extending mechanoreceptory setae to bend, which may elicit a response (Strickler \& Bal 1973, Yen et al. 1992). A velocity gradient may be decomposed in its rotational (vorticity) and irrotational (deformation rate) components. For deformation rate, one may further distinguish between pure 'longitudinal deformation rate', which is the fluid deformation in a velocity gradient characterised solely by gradients in the direction of the flow, and pure 'shear deformation', which is the fluid deformation in a simple shear flow (i.e. velocity gradients solely perpendicular to the flow direction). Velocity gradients obviously cause the fluid to accelerate. 
Each of these components and characteristics of a velocity gradient (vorticity, acceleration, longitudinal and shear deformation) cause a velocity difference between the copepod and the ambient fluid and may, thus, potentially be perceived (Kiorboe \& Visser 1999). Because the different components generate different signal strengths and attenuate differently from a moving predator (Kiørboe \& Visser 1999), it is of interest to both identify the component(s) that a copepod can perceive, and to determine threshold values for response.

Several previous studies have attempted to identify the components of the fluid disturbance to which a copepod reacts and to determine threshold values for reaction (Schröder 1967, Haury et al. 1980, Kirk \& Gilbert 1988, Fields \& Yen 1996, 1997 a, Heuch \& Karlson 1997). However, in most experimental (and real) situations, the different components co-vary, and it thus becomes difficult to identify the relevant one(s). Here we attempt to experimentally isolate one or a few components at a time, allowing us to identify the crucial ones and to determine threshold values. We observed copepods (Acartia tonsa) in different hydrodynamic devices (Fig. 1), a Couette tank (shear deformation, vorticity, acceleration), a rolling tank (vorticity, acceleration), in a flow generated by a suction pipette (longitudinal deformation, acceleration), and in an oscillating chamber (acceleration), and noted the minimum intensities of the fluid disturbance generated by these devices at which the copepod responded by escape reactions or increased jump frequencies.

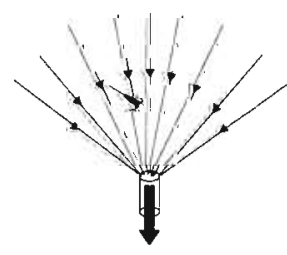

Siphon flow

- longitudinal deformation - acceleration

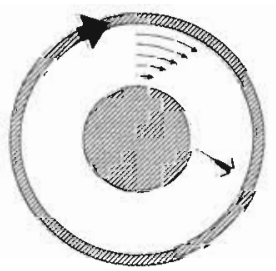

Couette device

- shear deformation

- acceleration

- vorticity

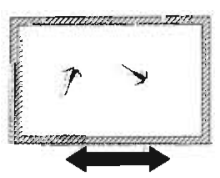

Oscillating chamber

- acceleration

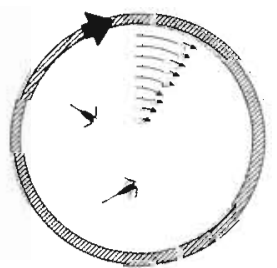

Rotating cylinder

- acceleration

- vorticity
Fig. 1 Schematic of the 4 hydrodynamic devices used to examine the responsiveness of Acartia tonsa to different components of a fluid disturbance
Our general hypothesis is that the threshold value for response to a particular component of a fluid disturbance depends solely on the magnitude of the velocity difference between the copepod and the ambient fluid that the fluid component generates (= signal strength). This hypothesis is based on the assumption that the copepod perceives fluid disturbances by means of mechanoreceptory setae that extend into the fluid; a velocity difference between the copepod and the ambient fluid causes these setae to bend, which may elicit an escape response (Strickler \& Bal 1973, Yen et al. 1992). Our hypothesis has implications for determining which components of a fluid disturbance generated by a real predator that a copepod responds to. It also has implications for the variation in responsiveness as a function of the size of the copepod, because the velocity difference due to a particular fluid disturbance generally increases with the size of the copepod, but in a different manner depending on the component considered. Below we first describe the fluid flow generated by our different hydrodynamic devices and quantify the velocity differences that they cause. On that basis we make the general hypothesis specific, quantifiable and testable. Subsequently we test the explicit hypotheses thus formulated.

\section{FLOW ANALYSIS}

\section{Suction flow (pure longitudinal deformation and acceleration)}

A suction siphon submerged in a fluid ideally generates along-flow velocity gradients in the ambient fluid (pure longitudinal deformation) and causes the fluid to accelerate $_{i}$ there is no shear deformation and no vorticity. If $r$ is the distance from the mouth of the siphon, $v$ the flow velocity and $Q$ the volume flow, then the flow velocity varies with distance as

$$
v(r)=-Q /\left(4 \pi r^{2}\right)
$$

The maximum deformation rate, $\Delta$, is the deformation rate along the length axis that yields the highest absolute value (cf. Kiørboe \& Visser 1999). This is here the radial axis and

$$
\Delta=\frac{Q}{2 \pi r^{3}}
$$

The magnitude of the acceleration in the direction of the flow is

$$
a_{r}=V(r) \frac{\partial v(r)}{\partial r}=-\frac{Q^{2}}{8 \pi^{2} r^{5}}
$$

By noting the distance at which a copepod reacts to the suction flow, one can estimate the response threshold for either the (longitudinal) deformation rate or the acceleration, but one cannot immediately decide to 
which of these two the copepod reacts. The maximum velocity difference (signal strength, $S$ ) between the copepod and the ambient fluid due to fluid deformation is $S_{\Delta}=\Delta \times L$ ( $L$ being the radius of the animal). The velocity difference due to acceleration is less than $a_{r}(\beta-1) / \alpha$ (Kiørboe \& Visser 1999), where

$$
\begin{gathered}
\alpha=\frac{9 \mu}{L^{2}\left(2 \rho_{p}+\rho_{\mathrm{f}}\right)} \\
\beta=\frac{3 \rho_{\mathrm{f}}}{2 \rho_{\mathrm{p}}+\rho_{\mathrm{f}}}
\end{gathered}
$$

and $\mu$ is the dynamic viscosity of the fluid and $\rho_{p}$ and $\rho_{f}$ the density of the particle and the fluid, respectively.

A potential problem in such a set-up is that in the boundary layer around the siphon, friction may be important and decelerate the flow. The thickness of this boundary layer $(\delta)$ - within which the flow deviates more than $1 \%$ of the free-stream fluid velocity can very roughly be estimated as $5 \sqrt{\frac{X v}{U}}$, where $X$ is the distance that the siphon extends into the fluid, $v$ is the kinematic viscosity $\left(-10^{-2} \mathrm{~cm}^{2} \mathrm{~s}^{-1}\right)$, and $U$ the freestream fluid velocity (Vogel 1994). At the mouth of the pipette, $U=Q / 4 \pi C^{2}(c=\text { siphon radius })_{\text {; }}$ hence $\delta \approx$ $8.9 c \sqrt{\frac{X v}{Q}}$. A combination of a narrow pipette and a high flow rate minimises the boundary layer thickness.

\section{Oscillating chamber (acceleration)}

Copepods in a closed chamber that accelerates will experience only acceleration; there are no velocity gradients and, hence, no deformation. If the chamber oscillates sinusoidally in one direction with amplitude $z_{0}$, frequency $\sigma(=2 \pi /$ period $)$ and velocity amplitude $u_{0}$ $\left(=z_{0} \sigma\right)$, then the velocity of the fluid $(u)$ is

$$
u(t)=z_{0} \sigma \sin \sigma t
$$

and the acceleration (a):

$$
a(t)=\frac{\mathrm{d} u}{\mathrm{~d} t}=u_{0} \sigma \cos \sigma t=z_{0} \sigma^{2} \cos \sigma t
$$

Thus, maximum acceleration is $z_{0} \sigma^{2}$.

Since the copepod has a density slightly higher than that of the ambient water, it 'slips' after the water in an accelerating fluid. The magnitude of this 'slip velocity' can be estimated as follows. The equation of motion for a rigid sphere moving with velocity $v$ in a fluid with velocity $u$ is (ignoring gravity, which is constant) (Maxey \& Riley 1983)

$$
\frac{\mathrm{d} v}{\mathrm{~d} t}=\alpha(u-v)+\beta \frac{\mathrm{d} u}{\mathrm{~d} t}
$$

For the oscillating case, Eq. (8) becomes

$$
\frac{\mathrm{d} V}{\mathrm{~d} t}+\alpha v=\beta u_{0} \sigma \cos \sigma t+\alpha u_{0} \sin \sigma t
$$

If $w(t)=v(t)-u(t)$ is the 'slip velocity' and $v=0$ at $t=0$, then this has a solution of the form

$$
\begin{aligned}
& w(t)=v(t)-u(t) \\
& =u_{0}(\beta-1) \frac{\sigma}{\sqrt{\alpha^{2}+\sigma^{2}}}\left(\cos (\sigma t-\phi)-\frac{\alpha}{\sqrt{\alpha^{2}+\sigma^{2}}} \mathrm{e}^{-\alpha t}\right)
\end{aligned}
$$

where $\tan (\phi)=\sigma / \alpha$. Thus, the maximum slip velocity (signal strength) is

$$
S_{\text {slip }}=u_{0}(\beta-1) \frac{\sigma}{\sqrt{\alpha^{2}-\sigma^{2}}}
$$

\section{Couette flow (acceleration, vorticity and pure shear deformation)}

A Couette device consists of 2 cylinders, one inside the other (Van Dureen 1968). In the annular gap between the 2 cylinders well-defined laminar shear (= velocity gradient perpendicular to the direction of the flow) and vorticity develops when one or both of the cylinders rotate. Thus, a combination is produced of acceleration, pure shear deformation and vorticity. For the outer cylinder of radius $I_{0}$ rotating at a rate $\Omega$ (rad $\mathrm{s}^{-1}$ ) and the inner cylinder (kept still) of radius $r_{i}$, the maximal deformation rate as a function of the radial distance, $r$, is given by

$$
\Delta=\Omega \frac{r_{0}{ }^{2} r_{i}{ }^{2}}{r^{2}\left(r_{0}{ }^{2}-r_{1}{ }^{2}\right)}
$$

which is the rate of shear deformation.

Maximum acceleration (at the periphery), which is only in the radial direction, is

$$
a_{r}=r_{0}^{2} \Omega
$$

Vorticity has 1 component in the axial ( $z$ ) direction

$$
\omega_{z}=2 \Omega \frac{r_{0}^{2}}{r_{0}^{2}-r_{j}^{2}}
$$

As above, the maximum velocity difference between the copepod and the ambient fluid due to deformation is given by the deformation rate multiplied by the radius of the animal. The velocity difference due to acceleration is $a_{r}=(\beta-1) / \alpha$ (Kiørboe \& Visser 1999), and the velocity difference due to vorticity is given below.

\section{Solid body rotation (vorticity)}

To generate vorticity, we rotated a closed cylinder around its own axis. After a short spin-up time, the fluid inside the cylinder rotates as a solid body (Jackson 1993). At a rotational velocity $\Omega\left(\mathrm{rad} \mathrm{s}^{-1}\right)$, the vorticity in the axial $(z)$ direction is given by 


$$
\omega_{z}=2 \Omega
$$

The velocity difference between the copepod and the ambient water due to vorticity can be estimated if we for simplicity assume that the copepod is spherical. If the sphere's centre of mass is identical to its buoyancy centre, then the sphere will exactly follow the solid body fluid rotation, and there will be no velocity difference. However, if the centre of mass is slightly offset from the geometrical centre (bottom-heavy), it will resume a specific orientation. The steady state orientation, $\xi^{\circ}$, is (Kiørboe \& Visser 1999, modified from Jonsson et al. 1991)

$$
\sin \xi^{*}=\frac{3 \mu \omega}{g l \rho_{\mathrm{p}}}
$$

where $l$ is the distance between the centre of buyancy and the centre of gravity, $\xi$ is the offset angle, and $g$ is gravitational acceleration. If the effect of rotation exceeds the effect of gravity, there will be no steady state orientation, and the particle will continue to rotate with the fluid (but at a rate less than that of the fluid). The critical vorticity $\left(\omega^{\circ}\right)$ for steady state orientation, i.e. when the effects of gravity and rotation balance is

$$
\omega^{*}=\frac{g l \rho_{p}}{3 \mu}
$$

For vorticities less the critical value, the particle will resume its steady state orientation and, thus, will not rotate with the fluid. The velocity difference (signal strength, $S_{\omega}$ ) can therefore be estimated as $1 / 2 L \omega$ (Kiørboe \& Visser 1999). For vorticities exceeding the critical value, the velocity difference does not exceed $1 / 2 L \omega^{\circ}$, irrespective of the actual magnitude of the vorticity.

Maximum acceleration (at periphery) and slip velocity are calculated as for the Couette device.

\section{Explicit hypotheses}

The above equations allow us to estimate the velocity difference between the copepod and the ambient fluid due to each of the flow components considered (pure longitudinal deformation, pure shear deformation, vorticity, acceleration) and in each of the flow generating devices. This, in turn, will allow us to test our general hypothesis, that an escape response is elicited by a threshold velocity difference (= signal strength), irrespective of how this is generated.

This hypothesis has a number of specific implications. First, because signal strength scales with copepod size if deformation rate provides the signal (whether it is longitudinal or shear deformation), we predict that threshold deformation rate declines with the inverse of the size of the animal provided threshold signal strength is constant. Likewise, if acceleration provides the signal, signal strength increases with the square of the linear dimension of the animal and, thus, threshold acceleration declines inversely with the square of the length of the copepod. Both cannot be correct at the same time and this difference in scaling provides a different means of assessing the significance of these 2 signals in suction flow experiments. Admittedly, these hypotheses are less rigid, because variation in size either means that different species or different developmental stages need be compared, and these do not necessarily have similar signal strength thresholds. Alternatively, comparing the responses of different developmental stages/different species is a way of comparing signal strength thresholds.

Finally, for the siphon experiment, it follows from Eqs. (2) \& (3) that reaction distance varies with $Q^{1 / 3}$ and $Q^{2 / 5}$ if deformation rate or acceleration, respectively, provide the signal. While this difference in exponents is likely too small to detect, examining variation in reaction distance with volume flow provides a test of the general concept of the siphon experiment.

\section{MATERIAL AND METHODS}

In all the experiments we used copepods from our laboratory culture of Acartia tonsa (Støttrup et. al 1986). Experiments were conducted at 18 to $20^{\circ} \mathrm{C}$. Behaviour was filmed by CCD cameras (Minitron MTV-1802CD) with 35 or $105 \mathrm{~mm}$ lenses and recorded on a Panasonic VCR (50 frames $\mathrm{s}^{-1}$ ) equipped with a video timer (1/100 s). Illumination was provided by infrared light emitting diodes and, unless otherwise specified, no white light was admitted.

Suction flow. Our experimental set-up was similar to that of Fields \& Yen (1996). A siphon-tube was extending from the centre of the bottom of a $25 \times 25 \times 50 \mathrm{~cm}^{3}$ aquarium, with the mouth $7 \mathrm{~cm}$ above the bottom. A $5 \mathrm{~mm}$ i.d. tube connected the outlet of the siphon with a flow regulator positioned $65 \mathrm{~cm}$ below the bottom of the aquarium. Escape responses to the flow generated by the siphon were recorded by 2 video cameras that were viewing the volume around the tip of the siphon at right angles and at a distance of 40 to $65 \mathrm{~cm}$. The 2 images were combined on 1 monitor with an image mixer and recorded. Escape distances were estimated from the $x-y-z$ coordinates thus recorded. We only considered escape events that occurred above the mouth of the siphon. During recordings we did not return water to the tank because that invariably generated turbulence. We rather allowed the water to sink in the tank, but by no more than $20 \mathrm{~cm}$. Because the flow in 
the outlet tube is turbulent, $Q$ scales with head ${ }^{0.5}$, and this $20 \%$ change in head (from 115 to $95 \mathrm{~cm}$ ) causes only a $10 \%$ change in volume flow and, thus, only a $\sim 2 \%$ variation in escape distance.

We measured flow velocities and recorded pathlines by following neutrally buoyant Artemia eggs (200 $\mathrm{mm}$ diameter) with frame-by-frame analyses of video recordings for the 2 different siphons used (i.d. 1.1 and $3.0 \mathrm{~mm}$ ) and within the range of volume flows applied ( $Q$ between 0.5 and $12 \mathrm{ml} \mathrm{s}^{-1}$ ).

We conducted 2 experimental series to measure escape distances. In Siphonl we quantified escape distances in adult females as a function of $Q$ for 5 values of $Q$ between 0.5 and $12 \mathrm{ml} \mathrm{s}^{-1}$. For these experiments we used the larger of the 2 pipettes (i.d. $3 \mathrm{~mm}$ ), had 600 to 800 females in the aquarium and analysed 12 to 65 escape responses per treatment. In SiphonII we followed how the escape response distances of a cohort of Acartia tonsa changed as the animals developed. For these experiments we used the smaller (1.1 $\mathrm{mm}$ i.d.) pipette and applied the same volume flow throughout (ca $1.4 \mathrm{ml} \mathrm{s}^{-1}$ ). About 200000 eggs spawned during $12 \mathrm{~h}$ were incubated in an $80 \mathrm{I}$ aerated tank at $18^{\circ} \mathrm{C}$. Every 2 to $3 \mathrm{~d}, 2000$ to 10000 nauplii or copepodites of almost identical developmental stage were transferred to the siphon-tank and about 40 escape events analysed. In one experiment adult females only were used. Animals were staged and sized every time.

Oscillating chamber. A closed experimental châmber $\left(65 \mathrm{ml}\right.$ Nunc culture flask, ca $2 \times 4 \times 8 \mathrm{~cm}^{3}$ ) was mounted on a sledge, which could be moved horizontally. A cylinder mounted eccentrically on a rotating axis generated sinusoidal motion. The eccentricity varied along the length of the cylinder, from 0 to $5 \mathrm{~mm}$. A small wheel was mounted on the sledge so that it touched the periphery of the rotating cylinder. Constant contact was secured by a spring that pressed the wheel of the sledge towards the cylinder, thereby transferring the sinusoidal motion to the sledge and the experimental chamber. By changing the point of contact between cylinder and sledge/wheel, the amplitude of the motion could be varied ( 0 to $2.5 \mathrm{~mm}$ ) : likewise, the speed of the motor could be adjusted, so that the frequency of oscillation could be varied between 0 and $10 \mathrm{~Hz}$. The behaviour of animals within the chamber was videorecorded from the side with the camera shutter set at short $(0.002 \mathrm{~s})$ exposure time to avoid a blurred picture.

We ran 2 experiments, one in which we used strong white illumination $(\mathrm{Oscl})$ and one in which illumination was provided by the infrared diodes (OsCII). In both experiments, 20 to 30 adult females were added to the chamber, and the chamber was oscillated at its maximum amplitude $(2.5 \mathrm{~mm})$ and consecutively at 0 .
$2,4,6,8$ and $10 \mathrm{~Hz}$. We filmed for $10 \mathrm{~min}$ at each frequency and allowed a break of $30 \mathrm{~min}$ in between. Videorecordings were subsequently analysed frameby-frame. 15 to 30 random individuals per treatment were followed for ca 1 to 20 s (until they disappeared from the screen) and the time of each jump recorded. We distinguished between short $(<2$ body lengths $)$ and long jumps. Frequencies of these 2 types of jumps as well as the frequency distribution of time intervals between jumps were used as response parameters.

Couette flow. Our Couette device is a copy of that described by van Duuren (1968); i.e. $I_{j}=4.35 \mathrm{~cm}, r_{0}=$ $5.65 \mathrm{~cm}$, length $24.9 \mathrm{~cm}, 1.01$ volume of the annular space, and only the outer cylinder rotating. For this design, flow is laminar up to at least $\Omega=12.5 \mathrm{rad} \mathrm{s}^{-1}$. For a given rotational velocity, the rate of shear deformation varies by a factor of ca 1.7 from near the inner to near the outer cylinder (cf. Eq. 12). The outer cylinder was made of transparent Plexiglas, thus allowing filming through the wall. The inner cylinder was black to increase the contrast.

We did 2 experiments, each time with ca 100 adult females in the chamber. In Couettel we applied rotational velocities of $0,0.12,0.28$, and $0.47 \mathrm{rad} \mathrm{s}^{-1}$, corresponding to average deformation rates of 0 to $0.93 \mathrm{~s}^{-1}$ and vorticities of 0 to $2.3 \mathrm{~s}^{-1}$. To better define the threshold value, Couettell was subsequently conducted with finer resolution, $\Omega=0,0.057,0.096,0.15$, 0.20 , and 0.24 rad $s^{-1}$, corresponding to deformation rates and vorticities of between $0-0.47$ and $0-1.2 \mathrm{~s}^{-1}$, respectively. We filmed 10 to $15 \mathrm{~min}$ at each setting and allowed breaks of $30 \mathrm{~min}$ between each. Videotapes were analysed for jump frequencies as above, except that the first 3 min was disregarded to allow the fluid flow to stabilise (Jackson 1993).

Solid body rotation. We used a simple cylindrical tube (inner diameter $=6 \mathrm{~cm}$, length $=8 \mathrm{~cm}$ ), closed with a stopper in one end and with a transparent Plexiglas wall in the other, as experimental chamber. This was placed on a 'rolling table' that consisted of 2 rotating bars that were driven by a motor with adjustable speed; this way the experimental chamber was rotated around its own length axis at rotational velocities, $\Omega$, of 0 to $5 \mathrm{rad} \mathrm{s}^{-1}$ or more. Filming was conducted through the transparent end wall.

We did experiments with about 50 aduit females in the chamber at $\Omega=0,0.3,0.6,1.3,2.5$, and $5.0 \mathrm{rad} \mathrm{s}^{-1}$ (and, thus vorticities twice that magnitude). Jump frequencies were analysed as above, again disregarding the first 3 min of rotation. In additional experiments (at $\Omega=0,0.7$, and $2.1 \mathrm{rad} \mathrm{s}^{-1}$ ), we estimated angular velocities of the copepods by estimating the orientation of non-swimming individuals at consecutive time intervals (typically $0.1 \mathrm{~s}$ intervals). In calm water the cope- 
pods jump and reorient frequently, but they will subsequently swing back towards a vertical position. By equating the gravitational torque with the torque required to rotate a particle at a certain angular velocity, $\mathrm{d} \xi / \mathrm{d} t$, it can be shown that (modified from Jonsson et al. 1991):

$$
\frac{\mathrm{d} \xi}{\mathrm{d} t}=\frac{g l \rho_{\mathrm{p}}}{6 \mu} \sin \xi+\Omega
$$

where $l$ is still the separation distance between the gravitational and the buoyancy centre and $g$ is the gravitational acceleration. Thus, the angular velocity relative to the fluid $\left(\frac{\mathrm{d} \xi}{\mathrm{d} t}-\Omega\right)$ is expected to be a sine function of the offset angle, both in calm and rotated water. The separation distance, l, and the critical vorticity can be estimated from the amplitude (Eqs. 15 \& 16).
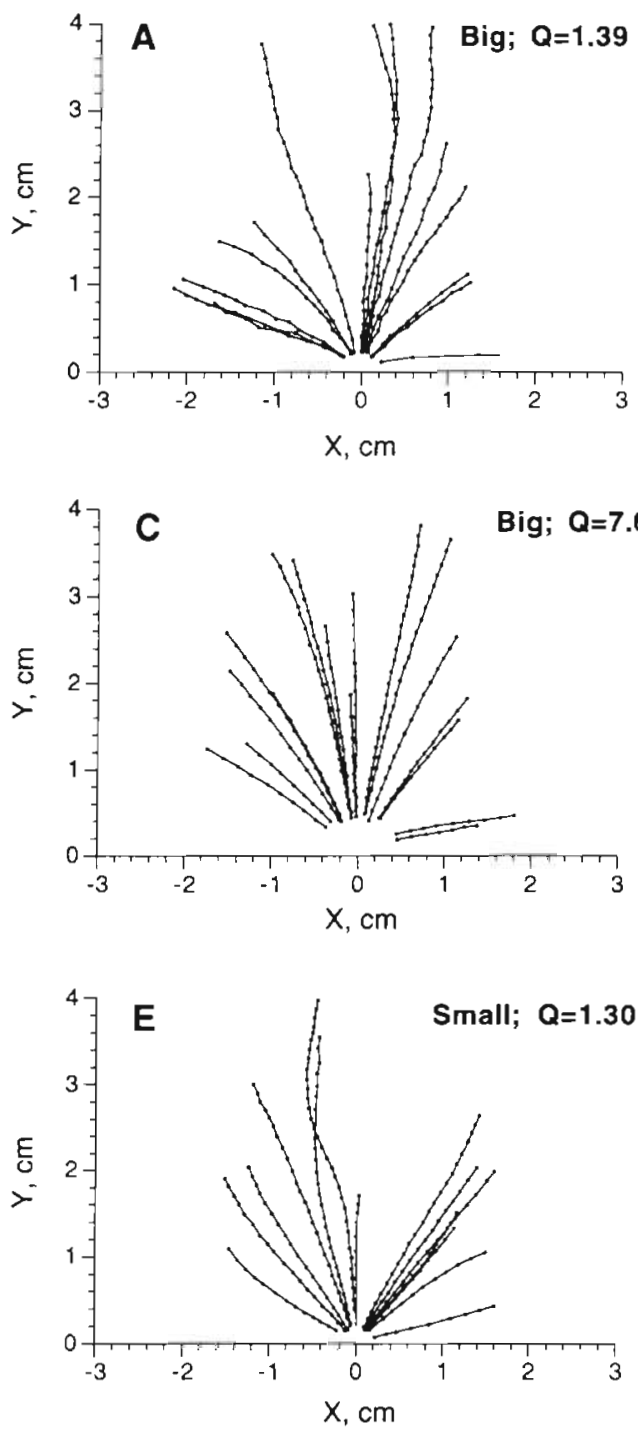

\section{RESULTS}

\section{Siphon flow}

Pathlines were radiating from the centre of the siphon opening and were approximately linear up to a distance of 10 to $20 \mathrm{~mm}$ from the mouth (Fig. 2). Beyond that distance, pathlines in several cases wiggled. Flow velocities varied approximately inversely with the square of the distance to the siphon opening, as predicted (Eq. 1, Fig. 3a-e). The volume flow estimated from fluid velocities and the regressions in Fig. 3a-e varied linearly with those measured directly by collecting water at the siphon outlet, but were about $17 \%$ higher (Fig. 3f). This can be accounted for by a small $(5 \%)$ calibration error. We therefore consider our calibration measurements consistent with the simple
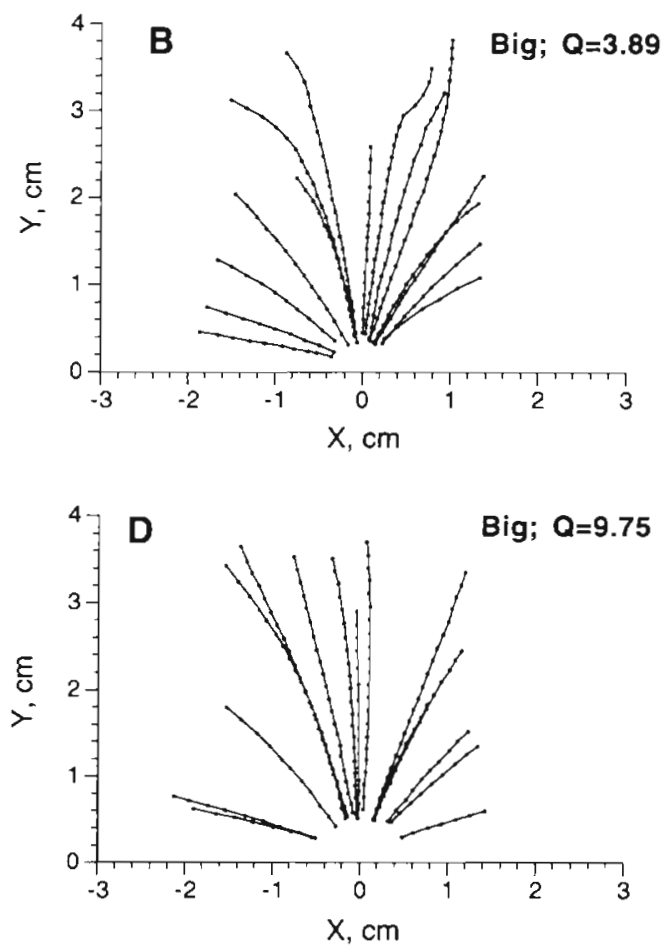

Fig. 2. Pathlines folded onto the $X-Y$ plane for selected volume flow rates $\left(Q, \mathrm{ml} \mathrm{s}^{-1}\right)$ of the 2 siphons used (Big; i.d. of $3.0 \mathrm{~mm}_{\mathrm{i}}$ Small: i.d. of $1.1 \mathrm{~mm}$ ). Each line represents the track of 1 particle over time, and the dots represent the position of the particle at various time intervals. We aimed at having equal distances rather than equal time intervals between particle positionings 

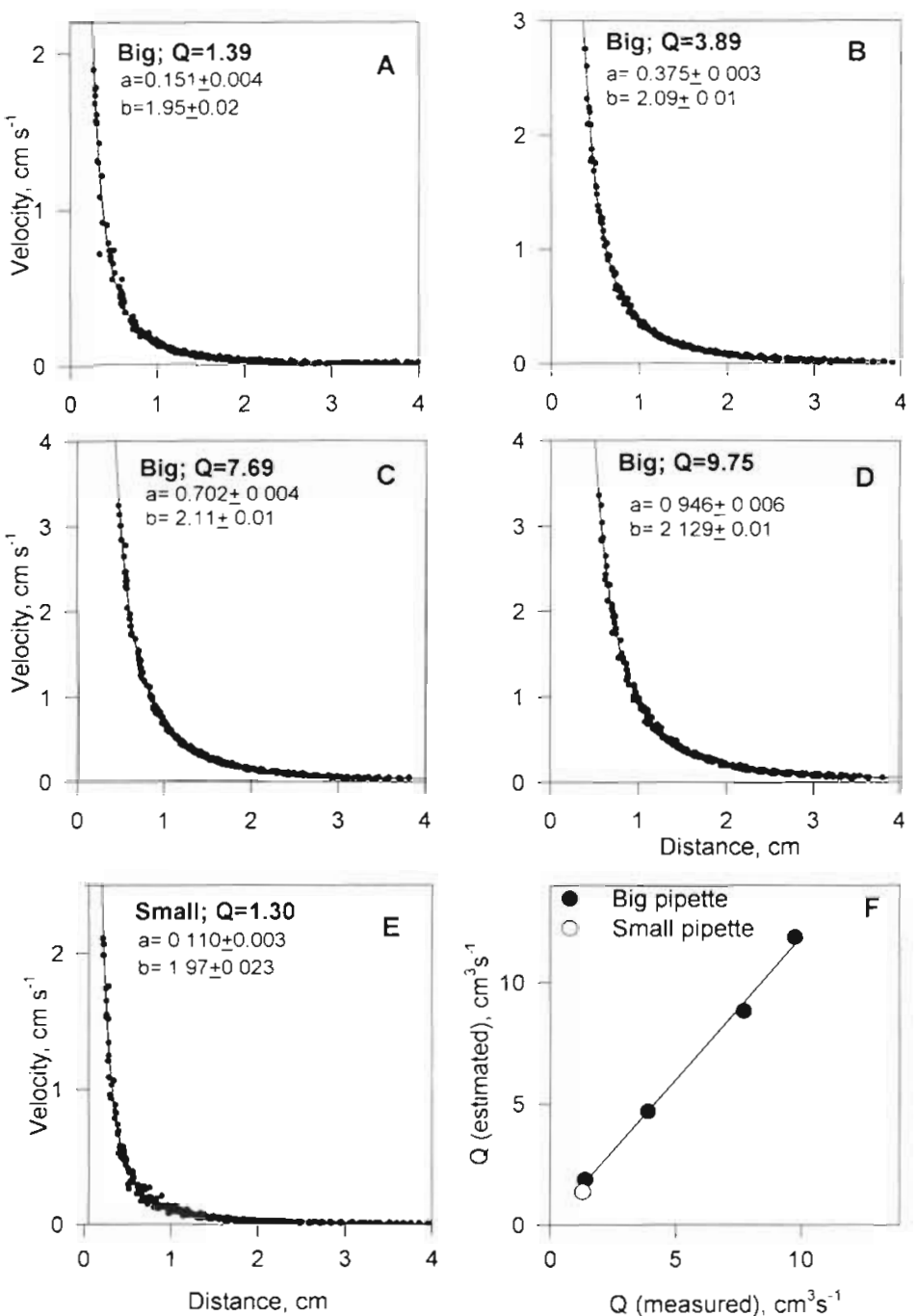

Fig. 3. (A-E) Fluid velocity $\left(v, \mathrm{~cm} \mathrm{~s}^{-1}\right)$ as a function of distance to the mouth of the siphon $(r, \mathrm{~cm})$ for selected volume flow rates $\left(Q, \mathrm{~cm}^{3} \mathrm{~s}^{-1}\right)$ for the 2 siphons used (Big: i.d. of $3.0 \mathrm{~mm}$ i Small: i.d. of $1.1 \mathrm{~mm}$ ). Nonlinear regressions of the form $v=a r^{-b}$ were fitted to the data, and the regression coefficients ( $a$ and $b \pm \mathrm{SD}$ ) are given for each configuration. (F) Comparison of volume flow rates estimated from the non-linear regressions (as $4 \pi \mathrm{a})\left(Q_{\mathrm{Est}}\right)$ and measured directly $\left(Q_{\mathrm{Mea}}\right), Q_{\mathrm{Est}}=$ $1.17 Q_{\text {Mea }}+0.15\left(r^{2}=0.996\right)$ flow model (Eqs. 1 \& 2), which was used in all subsequent calculations.

The copepods' reaction to the flow generated by the siphon was in many cases distinct: one or several consecutive long jumps that brought the copepod outside the flow field. In other cases the escape responses were less distinct and the copepod did not escape the flow in the first instance, but rather continued jumping against the flow, in some instances for several minutes. In almost all cases adult females managed to eventually escape the flow, while the nauplii's attempts to escape were not always successful. The reaction distance was in all cases defined as the distance at which the copepod first reacted.

Average reaction distances and the deformation rate (geometrical mean and $95 \%$ confidence intervals) and fluid acceleration at the point of escape are shown in Table 1 for the experiments in which the volume flow was varied (SiphonI). Reaction distances were all well outside the boundary layer. The calculated maximum slip velocities due to fluid acceleration are also shown. In calculating these slip velocities we assumed a density of Acartia tonsa $2.5 \%$ higher than that of the water and an equivalent spherical radius of an $A$. tonsa female of $0.023 \mathrm{~cm}$; the latter was estimated assuming a female carbon content of $5 \mu \mathrm{g} \mathrm{C}$ (Berggreen et al. 1988) and a carbon to volume

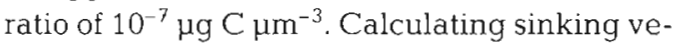
locity from Stokes' law on these assumption leads to an estimate that is ca 4 times higher than the actual sinking velocity $(0.28$ vs $0.07 \mathrm{~cm} \mathrm{~s}^{-1}$ ), and the calculated slip velocities are, thus, maximum estimates. These are, nevertheless, in all cases orders of magnitude less than the velocity difference due to fluid deformation rates $(\sim 0.5 \times$ length of the copepod $x$

Table 1. Acartia tonsa adult females in siphon flow. Average reaction distance $(R)( \pm S D)$ to mouth of siphon and calculated average acceleration and deformation rate (geometrical mean and $95 \%$ confidence interval) at the point of escape at various volume flow rates $(Q)$. Maximum slip velocity calculated by assuming copepods to have an equivalent spherical radius of $0.023 \mathrm{~cm}$ and a density $2.5 \%$ in excess of the ambient water. $\mathrm{N}$ is number of observations and boundary layer thickness is calculated as described in text

\begin{tabular}{|ccccccc|}
\hline $\begin{array}{c}Q \\
\left(\mathrm{ml} \mathrm{s}^{-1}\right)\end{array}$ & $N$ & $\begin{array}{c}R \\
(\mathrm{~cm})\end{array}$ & $\begin{array}{c}\text { Deformation rate } \\
\left(\mathrm{s}^{-1}\right)\end{array}$ & $\begin{array}{c}\text { Acceleration } \\
\left(\mathrm{cm} \mathrm{s}^{-2}\right)\end{array}$ & $\begin{array}{c}\text { Max slip } \\
\left(\mathrm{cm} \mathrm{s}^{-1}\right)\end{array}$ & $\begin{array}{c}\text { Boundary layer } \\
\left(\mathrm{cm}^{2}\right)\end{array}$ \\
\hline 0.50 & 21 & $0.757 \pm 0.127$ & $0.19(0.15-0.24)$ & 0.013 & $3.8 \times 10^{-6}$ & 0.50 \\
1.28 & 41 & $0.813 \pm 0.199$ & $0.42(0.33-0.54)$ & 0.058 & $1.7 \times 10^{-5}$ & 0.31 \\
5.13 & 36 & $1.295 \pm 0.248$ & $0.40(0.32-0.50)$ & 0.092 & $2.7 \times 10^{-5}$ & 0.15 \\
8.47 & 64 & $1.265 \pm 0.254$ & $0.71(0.61-0.83)$ & 0.28 & $8.2 \times 10^{-5}$ & 0.12 \\
11.58 & 41 & $1.409 \pm 0.307$ & $0.72(0.57-0.92)$ & 0.31 & $9.0 \times 10^{-5}$ & 0.10 \\
11.81 & 12 & $1.110 \pm 0.191$ & $1.43(1.17-1.76)$ & 1.05 & $3.1 \times 10^{-4}$ & 0.10 \\
\hline
\end{tabular}




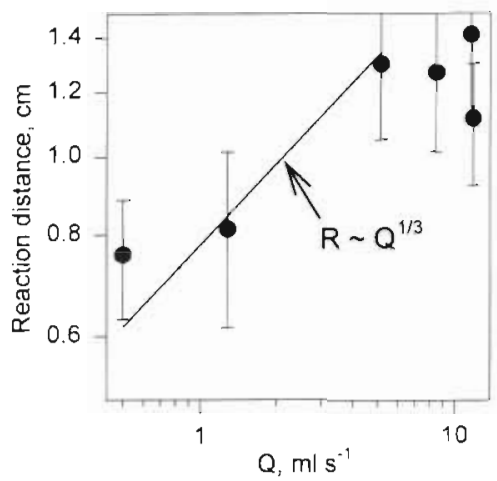

Fig. 4. Acartia tonsa in siphon experiment. Reaction distance $(R, \mathrm{~cm}$, measured from mouth of siphon) as a function of volume flow rate $\left(Q, \mathrm{~cm}^{3} \mathrm{~s}^{-1}\right)$. A cubic root regression is fitted to observations with $Q<6 \mathrm{ml} \mathrm{s}^{-1} ; R=0.778( \pm 0.049) Q^{1 / 3}$

deformation rate $\sim 0.5 \mathrm{~mm} \times$ deformation rate) at the point of escape. This was also the case in the experiment in which different developmental stages were tested (SiphonII, calculations not shown). If our hypothesis is correct, then in the siphon experiments it is the deformation rate rather than the fluid acceleration that generates the triggering signal. This does not necessarily imply, however, that Acartia tonsa is unable to perceive acceleration, only that signals due to acceleration can likely be disregarded in these experiments.

Reaction distance vs volume flow (Siphon $)$. The reaction distance generally increased with increasing volume flow, but only for low flows did it vary approximately with $Q^{1 / 3}$, as predicted (Fig. 4). Volume flows higher than $\sim 5 \mathrm{ml} \mathrm{s}^{-1}$ did not lead to much increased reaction distances. As a consequence, the estimates of threshold deformation rates were higher for the higher than for the lower volume flows. We are not sure whether this is due to non-linearity of pathlines at long distances (cf. Fig. 2), but the observation illustrates an

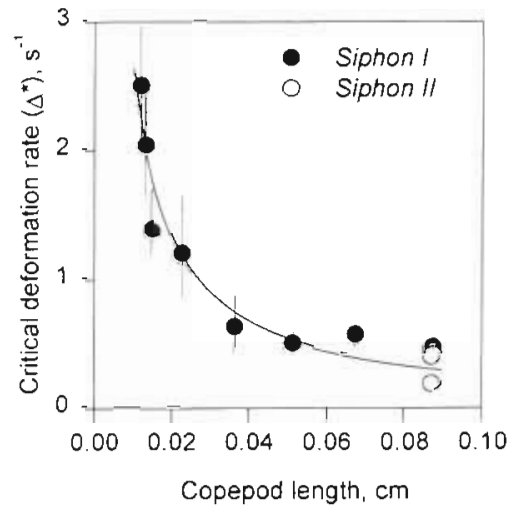

Fig. 5. Acartia tonsa in siphon experiment. Threshold deformation rate $\left(\Delta^{*}, 5^{-1}\right)( \pm 95 \% C L)$ versus size (prosome length, $\mathrm{cm}$ ) in individuals of different developmental stage and size. Open symbols are data from experiment SiphonI and closed symbols from SiphonII. A hyperbolic regression is fitted to the data of SiphonII: $\Delta^{*}=0.0264( \pm 0.0014) /$ length

apparent limitation of the siphon flow technique. In subsequent experiments we therefore used a lower $Q$.

Disregarding the estimates of critical deformation rate obtained at the higher volume flow rates, it appears that for Acartia tonsa females the threshold deformation rate for pure longitudinal deformation is on order 0.2 to $0.4 \mathrm{~s}^{-1}$ (Table 1). From the slope of the cubic root regression (including only the lower flow rates) relating reaction distance to volume flow (Fig. 4), one can calculate an average threshold deformation rate of $\Delta^{*}=0.34 \pm 0.06 \mathrm{~s}^{-1}$ (cf. Eq. 2)

Threshold deformation rate vs copepod size/developmental stage (SiphonII). The threshold deformation rate $\left(\Delta^{*}\right)$ estimated for the various developmental stages/sizes of Acartia tonsa in siphon experiments varied inversely with their length, as hypothesised (Table 2, Fig. 5). This suggests that the signal strength required to elicit an escape response (threshold signal strength, $S^{*}$ ) does not vary between developmental

Table 2. Acartia tonsa. Average reaction distance ( $\pm S D$ ) to mouth of siphon for different developmental stages in a siphon flow, and calculated acceleration and deformation rate (geometrical mean and $95 \%$ confidence interval) at the point of escape. In each experiment the 2 most abundant stages have been indicated (with the dominant one underlined), and the number in parentheses indicates their percentage contribution. Average body length and age (from eggs) also shown. Boundary layer thickness in this set-up is about $0.10 \mathrm{~cm}$ - all events were well beyond that distance

\begin{tabular}{|c|c|c|c|c|c|c|c|}
\hline $\begin{array}{c}Q \\
\left(\mathrm{ml} \mathrm{s}^{-1}\right)\end{array}$ & $\begin{array}{l}\text { Stage } \\
(\mathrm{s})\end{array}$ & $\begin{array}{c}\text { Average size } \\
(\mu \mathrm{m})\end{array}$ & $\begin{array}{l}\text { Age from egg } \\
\text { (h) }\end{array}$ & $\begin{array}{c}R \\
(\mathrm{~cm})\end{array}$ & $\begin{array}{l}\text { Deformation rate } \\
\qquad\left(\mathrm{s}^{-1}\right)\end{array}$ & $\begin{array}{l}\text { Acceleration } \\
\left(\mathrm{cm} \mathrm{s}^{-2}\right)\end{array}$ & $N$ \\
\hline 1.38 & NI-NII $(100)$ & $119 \pm 11$ & 24 & $0.453 \pm 0.088$ & $2.49(2.08-2.97)$ & 1.26 & 36 \\
\hline 1.41 & NI-NII $(100)$ & $132 \pm 10$ & 48 & $0.492 \pm 0.110$ & $2.03(1.66-2.48)$ & 0.87 & 44 \\
\hline 1.41 & NII--NIII $(96)$ & $149 \pm 20$ & 72 & $0.558 \pm 0.118$ & $1.38(1.18-1.69)$ & 0.47 & 36 \\
\hline 1.27 & NIV-NV (96) & $225 \pm 55$ & 144 & $0.597 \pm 0.297$ & $1.19(0.85-1.66)$ & 0.27 & 40 \\
\hline 1.46 & Cl-CII (85) & $361 \pm 86$ & 192 & $0.763 \pm 0.261$ & $0.62(0.43-0.91)$ & 0.10 & 30 \\
\hline 1.42 & CII-CIII (77) & $511 \pm 72$ & 240 & $0.787 \pm 0.148$ & $0.49(0.41-0.59)$ & 0.08 & 36 \\
\hline 1.39 & $\underline{\mathrm{CIV}}-\mathrm{CV}(71)$ & $673 \pm 96$ & 312 & $0.753 \pm 0.164$ & $0.56(0.45-0.69)$ & 0.10 & 41 \\
\hline 1.28 & Female $(100)$ & $871 \pm 29$ & $>400$ & $0.774 \pm 0.135$ & $0.46(0.37-0.56)$ & 0.075 & 25 \\
\hline
\end{tabular}


Table 3. Acartia tonsa females. Jump frequencies as a function of oscillation frequency in 2 experiments. Maximum acceleration and maximum slip velocities were calculated according to Eqs. (7) \& (11), respectively. Copepods were assumed to have an equivalent spherical radius of $0.023 \mathrm{~cm}$ and a density $2.5 \%$ in excess of the ambient water

\begin{tabular}{|c|c|c|c|c|c|c|}
\hline Expt no. /Frequency, $\mathrm{s}^{-1}$ & 0 & 2 & 4 & 6 & 8 & 10 \\
\hline OscI-jump frequency. $\mathrm{s}^{-1}$ & $1.21 \pm 1.03$ & $1.13 \pm 0.48$ & $0.85 \pm 0.54$ & $0.62 \pm 0.36$ & $1.00 \pm 1.14$ & $0.87 \pm 0.49$ \\
\hline OscII-jump frequency, $\mathrm{s}^{-1}$ & $1.43 \pm 0.71$ & $1.31 \pm 0.82$ & $1.26 \pm 1.11$ & $0.67 \pm 0.46$ & $0.98 \pm 0.61$ & $0.93 \pm 1.05$ \\
\hline Acceleration, $\mathrm{cm} \mathrm{s}^{-2}$ & 0 & 39 & 158 & 355 & 588 & 987 \\
\hline Slip velocity, $\mathrm{cm} \mathrm{s}^{-1}$ & 0 & 0.011 & 0.042 & 0.086 & 0.136 & 0.187 \\
\hline
\end{tabular}

stages. A hyperbolic relation was fitted to the data $\left(\Delta^{*}=\right.$ $k /$ Length) by non-linear regression, and the estimated coefficient $\left(k=0.0264 \pm 0.0014 \mathrm{~cm} \mathrm{~s}^{-1}\right)$ provides an estimate of twice the threshold signal strength, $S_{\Delta}{ }^{*} \approx$ $0.013 \mathrm{~cm} \mathrm{~s}^{-1}$. We note that the threshold deformation rate estimated for adult females in these experiments (Table 2 ) is consistent with that obtained above (Table 1).

\section{Oscillator}

In the 2 independent experiments we found the same response in jump frequency to variation in oscillation frequency of the experimental chamber, i.e. decreasing jump frequency with oscillation frequency increasing up to $6 \mathrm{~Hz}$, and subsequently a slight increase with a further increase in oscillation frequency (Table 3). However, this increase was statistically significant in only 1 experiment (OscII, KruskalWallis 1 -way ANOVA, $p=0.01$ ). The frequency of long jumps (escapes) was insignificant $\left(<0.1 \mathrm{~s}^{-1}\right)$. This unexpected response suggests that the copepods can perceive the acceleration, but that acceleration does not elicit escape responses (i.e. long jumps).

The maximum fluid acceleration applied in the oscillating chamber was similar to gravitational acceleration, and the estimated slip velocity at the highest acceleration was $\sim 0.2 \mathrm{~cm} \mathrm{~s}^{-1}$ (Table 3 ). Because the slip velocities were calculated on the same assumptions of shape and density as above, they are likely overestimated by a factor of ca 4 . Even then, slip velocities at oscillation frequencies of $6 \mathrm{~Hz}$ and more are substantially higher than the velocity difference of $0.013 \mathrm{~cm} \mathrm{~s}^{-1}$ that elicited escape responses in the siphon experiment. Thus, these observations are inconsistent with the overall hypothesis that escape responses are elicited by a threshold velocity difference, independent of what causes it.

\section{Solid body rotation}

In still water, the rate at which non-swimming Acartia tonsa females swing back towards vertical position
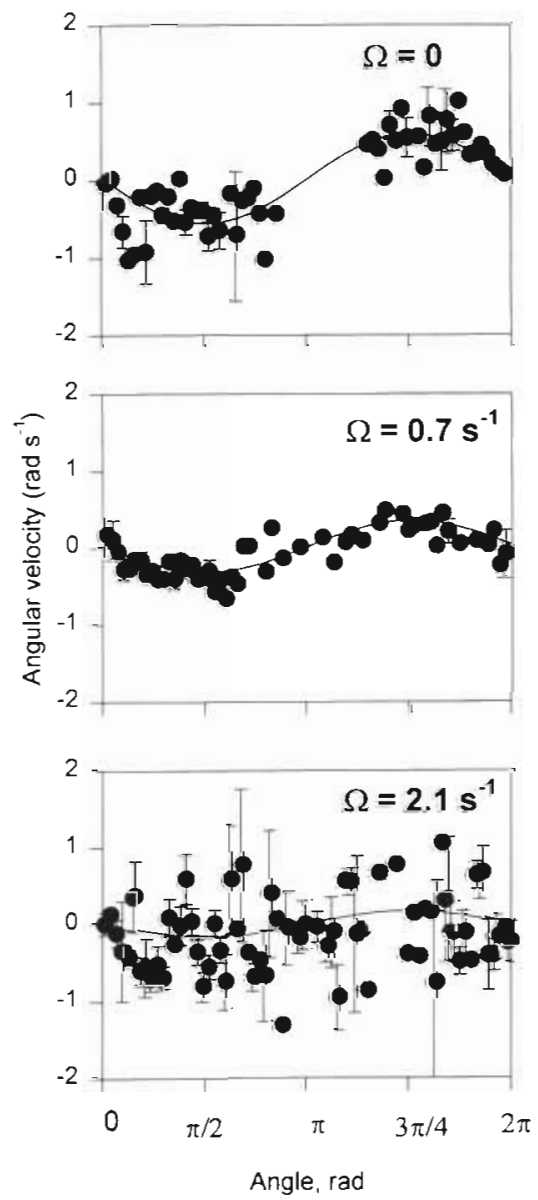

Fig. 6. Acartia tonsa in rotated water. Rotation velocity relative to fluid of adult females in calm and rotated water. Data binned in $5^{\circ}$ intervals. Sine functions are fitted to the data. Estimated amplitudes are $0.58 \pm 0.05 \mathrm{~s}^{-1}$ (at $\Omega=0$ ), $0.33 \pm 0.03$ $\mathrm{s}^{-1}\left(\right.$ at $\left.\Omega=0.7 \mathrm{~s}^{-1}\right)$, and $0.17 \pm 0.09 \mathrm{~s}^{-1}$ (at $\Omega=2.1 \mathrm{~s}^{-1}$ )

subsequent to a jump is - as expected - a sine function of the off-set angle (Fig. 6). The amplitude of the sine function, $0.58 \pm 0.05 \mathrm{~s}^{-1}$, suggests that the separation distance between the centres of buoyancy and gravity is only about $0.4 \mu \mathrm{m}$ and the critical vorticity for tumbling, $\omega^{*}$, is $-1.2 \mathrm{~s}^{-1}$. Thus, the maximum signal strength due to vorticity for a female copepod (length $\sim 0.09 \mathrm{~cm}$ ) is about $0.05 \mathrm{~cm} \mathrm{~s}^{-1}$. 
Table 4. Acartia tonsa adult females in rotating bottle. Total jump frequency (long + short jumps) ( \pm SD) as a function of rotational velocity $\left(\Omega\right.$, rad $\left.s^{-1}\right)$ and vorticity $\left(\omega_{z}\right)$

\begin{tabular}{|c|c|c|c|c|c|c|}
\hline$\Omega\left(\mathrm{s}^{-1}\right)$ & 0.0 & 0.3 & 0.6 & 1.3 & 2.5 & 5.0 \\
\hline$\omega_{z}\left(s^{-1}\right)$ & 0.0 & 0.6 & 1.2 & 2.6 & 5.0 & 10.0 \\
\hline Jump frequency $\left(s^{-1}\right)$ & $1.75 \pm 1.75$ & $1.41 \pm 0.93$ & $1.04 \pm 0.55$ & $1.50 \pm 0.60$ & $0.89 \pm 0.72$ & $1.14 \pm 0.99$ \\
\hline
\end{tabular}

The angular velocity of the copepod relative to the fluid also followed a sine function in a rotating fluid (Fig. 6). At $\Omega=0.7 \mathrm{~s}^{-1}$, where the vorticity $\left(\omega=1.4 \mathrm{~s}^{-1}\right)$ is close to the critical value, the amplitude of the oscillation is less than that in still water. This unexpected result may be due to violation of the assumption of sphericity. Using the amplitude of the sine function at this vorticity would suggest that the maximum signal strength due to vorticity for an adult female is rather $0.03 \mathrm{~cm} \mathrm{~s}^{-1}$. At the highest rotational velocity tested $(\Omega=$ $2.1 \mathrm{~s}^{-1}$ ), data are very noisy and the pattern difficult to see; this is likely because the rotational velocity of the copepod is small relative to the rotational velocity of the fluid and, hence, yielding a bad signal to noise ratio.

Maximum acceleration (at the periphery of the bottle) and maximum slip velocity increased to $45 \mathrm{~cm} \mathrm{~s}^{-2}$ and $0.013 \mathrm{~cm} \mathrm{~s}^{-1}$, respectively, at the highest rotational speed. This is less than that achieved in the oscillating chamber

The frequency of jumps varied erratically but significantly between treatments (Kruskal-Wallis 1-way analysis of variance, $p<0.05$; Table 4). However, only the treatment with a rotational speed of $2.5 \mathrm{rad} \mathrm{s}^{-1}$ differed significantly from the control (Dunn's test, p < 0.05). The frequency of long jumps was insignificant at all treatments and the observed pattern in jump frequency was inconsistent with escape responses. Thus, apparently, Acartia tonsa does not respond to the velocity difference generated by vorticity by escaping. Since the velocity difference estimated $\left(\sim 0.03 \mathrm{~cm} \mathrm{~s}^{-1}\right)$ is well above that estimated for pure longitudinal deformation, this observation is inconsistent with our general hypothesis.

\section{Couette flow}

The 2 independent experiments showed the same result: constant jump frequency with the initial increase in rotational speed of the Couette, and then an increase to a new level of jump frequencies (Fig. 7). Total and long jumps showed the same pattern, and the variation in the frequency of both types of jumps was statistically significant in both experiments (Kruskal-Wallis 1-way ANOVA, p < 0.05). We consider that the transition between low and high jump frequency demarcates the threshold. This threshold cor-
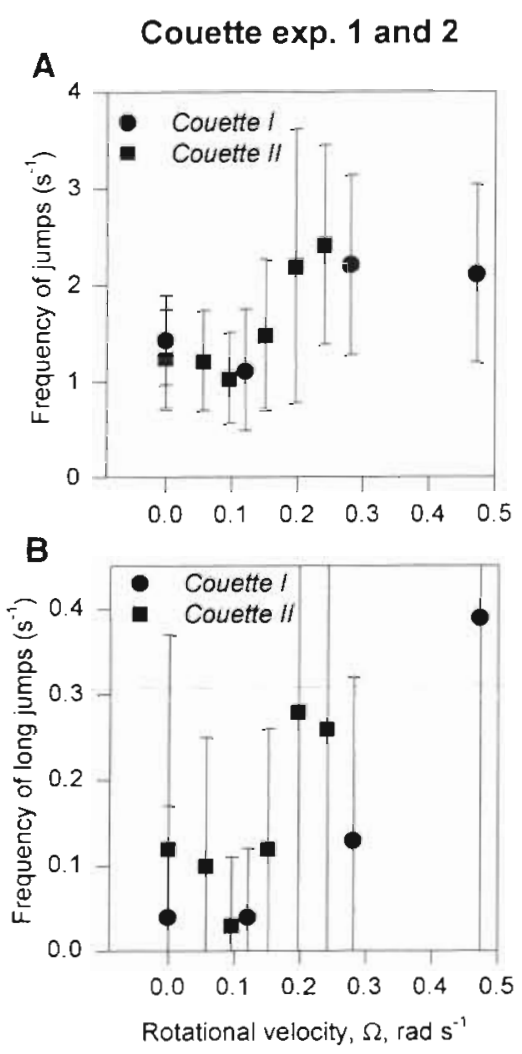

Fig. 7. Acartid Lonsa in Couette tank. (A) Total jump frequency and $(B)$ frequency of long jumps ( \pm SD) as a function of rotation velocity

responds to a rotational velocity, $\Omega$, of $0.20 \mathrm{rad} \mathrm{s}^{-1}$. At this rotational velocity the average shear deformation rate is $0.39 \mathrm{~s}^{-1}$ (range 0.29 to $0.48 \mathrm{~s}^{-1}$ ), the vorticity is $0.98 \mathrm{~s}^{-1}$, and maximum slip velocity due to acceleration is $0.002 \mathrm{~cm} \mathrm{~s}^{-1}$. We can disregard any signal due to vorticity and acceleration (cf. above), and the critical shear deformation rate estimated here is thus very similar to the critical longitudinal deformation rate estimated for adult females in the siphon experiment.

\section{DISCUSSION}

\section{Perception of velocity gradients}

A swimming predator may generate several types of water-born hydrodynamical signals, such as high fre- 
Table 5. Summary of siphon experiments reported in the literature. Threshold deformation rates were calculated from Eq. (2) on the basis of reported reaction distances and volume flows. Copepod sizes are prosome lengths

\begin{tabular}{|c|c|c|c|c|c|c|}
\hline Species & $\begin{array}{l}\text { Size } \\
(\mathrm{cm})\end{array}$ & $\begin{array}{c}Q \\
\left(\mathrm{ml} \mathrm{s}^{-1}\right)\end{array}$ & $\begin{array}{l}\text { Reaction } \\
\text { distance } \\
(\mathrm{cm})\end{array}$ & $\begin{array}{c}\text { Threshold } \\
\text { deformation rate } \\
\left(\mathrm{s}^{-1}\right)\end{array}$ & $\begin{array}{l}\text { Threshold } \\
\text { signal strength } \\
\left(\mathrm{cm} \mathrm{s}^{-1}\right)\end{array}$ & Source \\
\hline Euchaete rimana & 0.25 & 25 & $1.18 \pm 0.62$ & 2.4 & 0.3 & Fields \& Yen (1997a) \\
\hline Pleuromamma xiphias & 0.55 & 25 & $0.95 \pm 0.40$ & 4.6 & 1.3 & - \\
\hline Labidocera madurae & 0.30 & 25 & $0.86 \pm 0.52$ & 6.3 & 1.0 & - \\
\hline Acartia tonsa, adult & 0.08 & 0.053 & $0.28 \pm 0.19$ & 0.38 & 0.02 & - \\
\hline Acartia tonsa, nauplii. & 0.02 & 0.053 & $0.11 \pm 0.03$ & 6.3 & 0.06 & - \\
\hline Oithona sp. & 0.07 & 0.053 & $0.13 \pm 0.06$ & 3.8 & 0.13 & - \\
\hline Eurytemora affinis & 0.1 & 1.75 & $0.53 \pm 0.20$ & 1.9 & 0.19 & Vi.itasalo et al. (1998) \\
\hline Temora longicornis & 0.1 & 1.75 & $0.35 \pm 0.11$ & 6.5 & 0.65 & - \\
\hline Polyathra remata (Rotifer) & & $9.2 \times 10^{-4}$ & $0.042 \pm 0.003$ & 2.0 & - & Kirk \& Glibert (1988) \\
\hline Polyathra remata (Rotifer) & & $3.1 \times 10^{-3}$ & $0.054 \pm 0.003$ & 3.1 & - & - \\
\hline Calanus finmarchicus ${ }^{\star}$ & 0.25 & - & -. & 0.4 & 0.10 & Haury et al. (1980) \\
\hline
\end{tabular}

quency vibrations generated by oscillating appendages, high Reynolds number vortex shedding, far-field sound, etc., that may all be significant for predator detection (e.g. Yen \& Strickler 1996). In this study we consider only the responsiveness of copepods to the near-field, laminar velocity gradients that a body moving through water generates (Kiørboe \& Visser 1999).

We shall first review previous attempts to identify the component(s) of the flow field that elicits an escape response in copepods. Several authors have examined the response of zooplankters to siphon flows (e.g. Singarajah 1969, 1975, Landry 1978, Kirk \& Glibert 1988, Fields \& Yen 1996, Fields \& Yen 1997a, Viitasalo et al. 1998; Table 5), most notably and thoroughly Fields \& Yen (1996). These last-mentioned authors attempted to utilise the different spatial distribution of 'shear' (defined as the component of the velocity gradient perpendicular to the vertical direction) and acceleration to argue that the copepod Pleuromama xiphias responded to 'shear' rather than acceleration. However, their definition of 'shear' makes its magnitude and spatial distribution dependent on the orientation of the coordinate system to which the observations are referred and, as noted elsewhere (Viitasalo et al. 1998), their data interpretation is flawed. Haury et al. (1980) generated a fluid disturbance in a laminar flow by placing an obstacle in the flow; they noted the distance at which copepods (Calanus finmarchicus) in the flow responded to the obstacle. In this set-up, acceleration, fluid deformation and vorticity co-vary. The horizontal component of vorticity in this system is, however, zero, and vorticity thus cannot be sensed (Kiørboe \& Visser 1999). Haury et al. (1980) disregarded acceleration, because slip velocities were much less than the signal strength due to deformation; this is not a completely valid argument, though, and actual experiments are required. Schröder (1967) conducted a conceptually elegant pilot experiment that suggests that Cyclops copepodites can sense acceleration: copepods were placed in a water-filled glass tube in a car, and the car decelerated. This causes negative acceleration of the fluid but no velocity gradient. The applied acceleration (380 $\mathrm{cm} \mathrm{s}^{-2}$ ) would impose a slip velocity of about $1.3 \mathrm{~cm} \mathrm{~s}^{-1}$. In the more elaborate experiments of Schröder (1967), a velocity gradient was established to generate (negative) acceleration, and these are therefore inconclusive. Subsequent experiments by Heuch \& Karlson (1997) in an oscillating closed chamber have demonstrated that much lower accelerations, $\sim 1 \mathrm{~cm}$ $\mathrm{s}^{-2}$, yielding slip velocities of a few $\mu \mathrm{m} \mathrm{s}^{-1}$, may elicit a response (enhanced motility) in the planktonic copepodids of a parasitic copepod.

The present study is the first in which all the different components of laminar velocity gradients have been efficiently separated. Our observations do not fully support the general hypothesis erected in the introduction that the threshold intensity of a particular velocity gradient component depends solely on the signal strength (velocity difference) that it causes. As regards deformation rate, the estimated threshold for Acartia tonsa females was about $0.4 \mathrm{~s}^{-1}$, independent of whether the copepod experienced pure longitudinal or pure shear deformation, consistent with the hypothesis. This supports theoretical arguments that the orientation of the main axis of deformation relative to the flow direction plays no role in its detection (Kiørboe \& Visser 1999). This is different from chemical detection of prey in a feeding current, which is enhanced by a shear deformation component in the feeding current (Andrews 1983). The variation in estimated threshold values for 


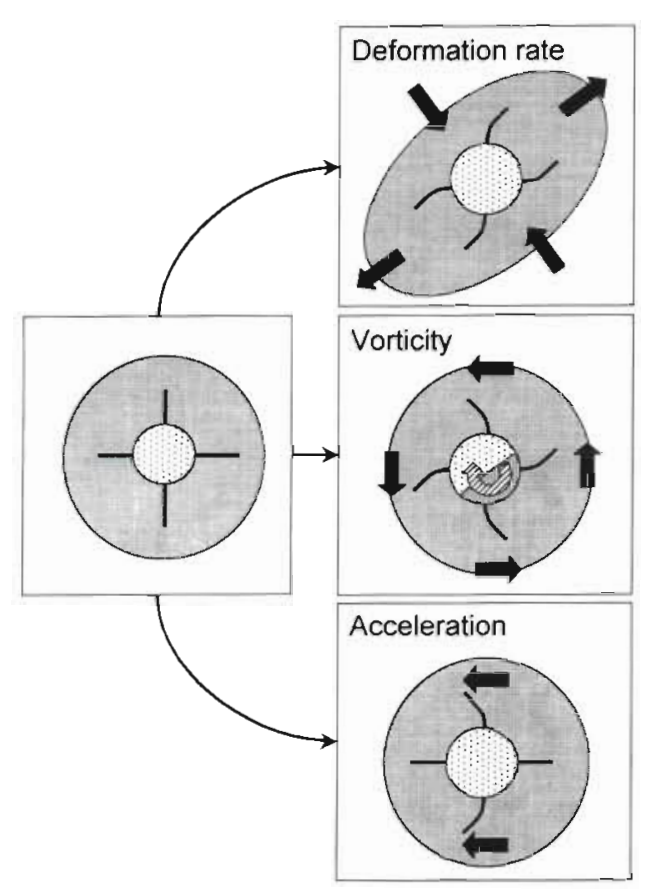

Fig. 8. Schematic describing how deformation, vorticity and acceleration cause different bending patterns of the setae distributed over the body of a copepod. The bending directionof a setae depends on its orientation

deformation rate with variation in copepod size is consistent with a set threshold signal strength, also as hypothesised. Vorticity and acceleration, on the other hand, did not elicit escape responses, even though the generated velocity differences (signal strengths) considerably exceeded that due to threshold intensity of deformation rate. However, deformation, vorticity and acceleration causes different setal bending patterns (Fig. 8) and may, hence, be distinguished by the copepod. Applied acceleration, for example, causes a uniform velocity difference across the entire body of the copepod (as does swimming and sinking), and such a signal may be interpreted differently by the multiple setae distributed over the body of the animal than signals due to vorticity and deformation, where the induced velocity difference varies across the body. A. tonsa females are able to perceive acceleration, as judged from the response in jump frequency (Table 3), but their response cannot be interpreted as escape attempts and its ecological significance is unclear. Signals due to vorticity resemble those due to undisturbed gravitationally induced 'rotation' behaviour of a copepod and the signal strength due to applied vorticity is similar to or less than that due to normal reorientation behaviour (Fig.6). Hence, A. tonsa may not be able to distinguish the signals due to vorticity from those due to normal behaviour. That vorticity cannot be perceived by $A$. tonsa is not a trivial result, and does not necessar- ily apply generally. Copepods, or other organisms that depend on mechanoreceptory setae to perceive hydrodynamical disturbances, and which have a more constant orientation when undisturbed, may have such capability. We conclude that $A$. tonsa is capable of distinguishing between signals of similar magnitude that are due to different components of a fluid disturbance, presumably by means of the array of setae that are distributed all over the body of the copepod. Deformation, irrespective of its orientation, is the component of a velocity gradient that most generally elicits escape in copepods; vorticity may be perceived by organisms with a constant orientation (remains to be demonstrated, though), while available evidence suggests that acceleration, although sensed, may not elicit escapes.

\section{Magnitude of signal strength}

Siphon and Couette experiments together suggest that the threshold signal strength for escapes in Acartia tonsa is about $0.015 \mathrm{~cm} \mathrm{~s}^{-1}$ and that it is invariant with developmental stage. Yen \& Fields (1992) characterised the flow field at the point of escape of nauplii of Acartia hudsonica entrained in the feeding current of Temora longicornis and estimated the (difficult to measure) velocity difference between the prey and the ambient fluid. The average $( \pm S D)$ velocity difference from their observations, $0.03 \pm 0.03 \mathrm{~cm} \mathrm{~s}^{-1}$, is of the same order as that estimated from their observed flow characteristics (Kiørboe \& Visser 1999), $0.015 \mathrm{~cm} \mathrm{~s}^{-1}$, and similar to that observed here for $A$. tonsa. Further, estimates of threshold signal strength in $A$. tonsa, calculated from the siphon experiments of Fields \& Yen (1997a) by applying Eq. (2) (Table 5), are of the same order as those found here, and the several independent estimates thus fall within a relatively narrow range. Table 5 also compiles reinterpretations of siphon experiment data for other copepod species, and together with our data demonstrates that threshold signal strengths vary by almost 2 orders of magnitude $\left(0.013\right.$ to $\left.1.3 \mathrm{~cm} \mathrm{~s}^{-1}\right)$, while estimated threshold deformation rates vary considerably less, 0.4 to $6.3 \mathrm{~s}^{-1}$. For comparison, estimates of threshold deformation rate of a rotifer have also been included (this nonrigid organism likely perceives deformation more directly and by a mechanism different from that of copepods; hence no threshold signal strength calculated), and this is of the same order. Thus, threshold deformation rate varies relatively little between species.

\section{Ecological significance}

Estimated threshold signal strengths may be compared with electrophysiological observations. Yen et al. 
(1992) found that antennal setae bending at a rate of as little as $20 \mu \mathrm{m} \mathrm{s}^{-1}$ was sufficient to elicit a neurophysiological response in Labidocera madurae. This is less by several orders of magnitude the threshold signal strength required to elicit escape responses in copepods (Table 5). What causes this difference between potential and realised behavioural threshold and the remarkable narrow variation in response thresholds between species? We propose that the behavioural response threshold represents the compromise between avoidance of inordinate escapes due to turbulent fluid deformation (cf. Fields \& Yen 1997a) and the responsiveness required to detect predators. We qualify this below.

Small-scale turbulence elicits escape responses in copepods (Costello et al. 1990, Saiz \& Alcaraz 1992). Fluid deformation rates due to upper ocean turbulence are up to $1-10 \mathrm{~s}^{-1}$ (reviewed in Kiørboe \& Saiz 1995) and the thresholds reported here for Acartia tonsa and other copepods are thus similar to maximal turbulent deformation rates in the ocean. Contrary to Fields \& Yen (1997a), we see no clear relation between deformation thresholds as calculated in Table 5 and maximum turbulent intensities of the habitats of the different species, mainly because peak turbulence in the upper ocean is determined primarily by winds. During windy condition, adult Acartia for example, residing near the surface, may experience turbulent deformation rates exceeding their response threshold. To prevent interference with predator (and prey) detection, copepods may respond to periods of elevated wind generated turbulence by descending in the water column (Mackas et al. 1993), by concentrating at depth strata of minimal turbulence (e.g. pycnoclines), or by residing permanently at depth.

The sensitivity of copepods to fluid deformation as recorded in Table 5 appear to be exactly sufficient to make simple cruise predator or feeding current strategies inefficient in capturing copepods. Simple examples may serve to illustrate this. The predatory copepod Centropages hamatus generates a feeding current of a maximal velocity $(U)$ of $0.08 \mathrm{~cm} \mathrm{~s}^{-1}$ and has a capture volume (within which the feeding current is generated) of radius (C) of $0.025 \mathrm{~cm}$ (Fig. 1 of Tiselius \& Jonsson 1990). From these values, the copepod feeding current model of Kiørboe \& Visser (1999) predicts the attenuation of the feeding current velocity directly in front of the Centropages, and this accords well with observations (Fig. 9a). Acartia nauplii entrained in this feeding current escape at an average distance of $0.092 \mathrm{~cm}$ (Tiselius \& Jonsson 1990). The threshold deformation rate and threshold signal strength of Acartia stage I nauplii (Table 2) and the modelled attenuation of deformation rate would predict a similar escape distance directly in front, about $0.11 \mathrm{~cm}$ (Fig. 9b,c), of the predatory copepod and slightly closer if the copepod arrives more laterally in the feeding current. Nauplii escape velocity (1 to $2 \mathrm{~cm} \mathrm{~s}^{-1}$, unpubl. obs.) exceeds several-fold the feeding current velocity at this distance (ca $0.25 \mathrm{~cm} \mathrm{~s}^{-1}$ ), and is in fact everywhere higher than the feeding current velocity, and the nauplius may thus escape. Less sensitive species will perceive the feeding current somewhat closer to the predatory copepod, but even here typical escape velocities $\left(\sim 100\right.$ body length $\mathrm{s}^{-1}$, see review by Mauchline 1998) exceed the feeding current velocity, thus allowing escape. Only copepods with threshold deformation rates exceeding the peak maximum deformation rate of the feeding current $(=8 U / 3 c$, Kiørboe \& Visser 1999), $12 \mathrm{~s}^{-1}$, will never perceive the feeding current (cf. Fig. 9b). More generally, since maximum feeding current velocity scales approximately with size in copepods, peak maximum deformation rates are rel-

Table 6. Summary of size (cephalothorax length and width), maximum feeding current velocity and estimated peak maximum deformation rate $\left(\Delta_{\max }\right)$ of the feeding current in representative copepods. Peak maximum deformation rate estimated as $3 U / 8 \mathrm{C}$, where $c$ is the radius of the capture volume (Kiørboe \& Visser 1999). $c$ was assumed to be $2 / 3$ of cephalothorax width (consistent with measurements in Eucalanus crassus and E. pileatus; Strickler 1985, Paffenhöfer \& Lewis 1990)

\begin{tabular}{|lccccc|}
\hline Species & Length & Width & $\begin{array}{c}\text { Max. feeding } \\
\text { curent velocity } \\
\left(\mathrm{cm} \mathrm{s}^{-1}\right)\end{array}$ & $\begin{array}{c}\text { Peak maximum } \\
\text { deformation rate } \\
\left(\mathrm{s}^{-1}\right)\end{array}$ & Source \\
\hline Eucalanus pileatus & 0.2 & 0.07 & 0.6 & 4.8 & Paffenhöfer \& Lewis (1990) \\
E. crassus & 0.18 & 0.06 & 1.0 & 9.3 & Strickler (1985) \\
E. rimana & 0.25 & 0.09 & 0.9 & 5.6 & Fields \& Yen (1997b) \\
Pleuromamma xiphias & 0.55 & 0.20 & 3.8 & 10.7 & Fields \& Yen (1993) \\
Paracalanus parvus & 0.067 & 0.023 & 0.4 & 9.8 & Tiselius \& Jonsson (1990) \\
Pseudocalanus elongatus & 0.092 & 0.030 & 0.5 & 9.4 & - \\
Temora longicornis & 0.097 & 0.044 & 0.8 & 10.2 & - \\
Centropages typicus & 0.13 & 0.041 & 0.8 & 11.0 & - \\
Centropages hamatus & 0.096 & 0.036 & 1.0 & 15.6 & Jonsson \& Tiselius (1990) \\
Acartia tonsa & 0.085 & 0.027 & 0.8 & 16.7 & \\
\hline
\end{tabular}



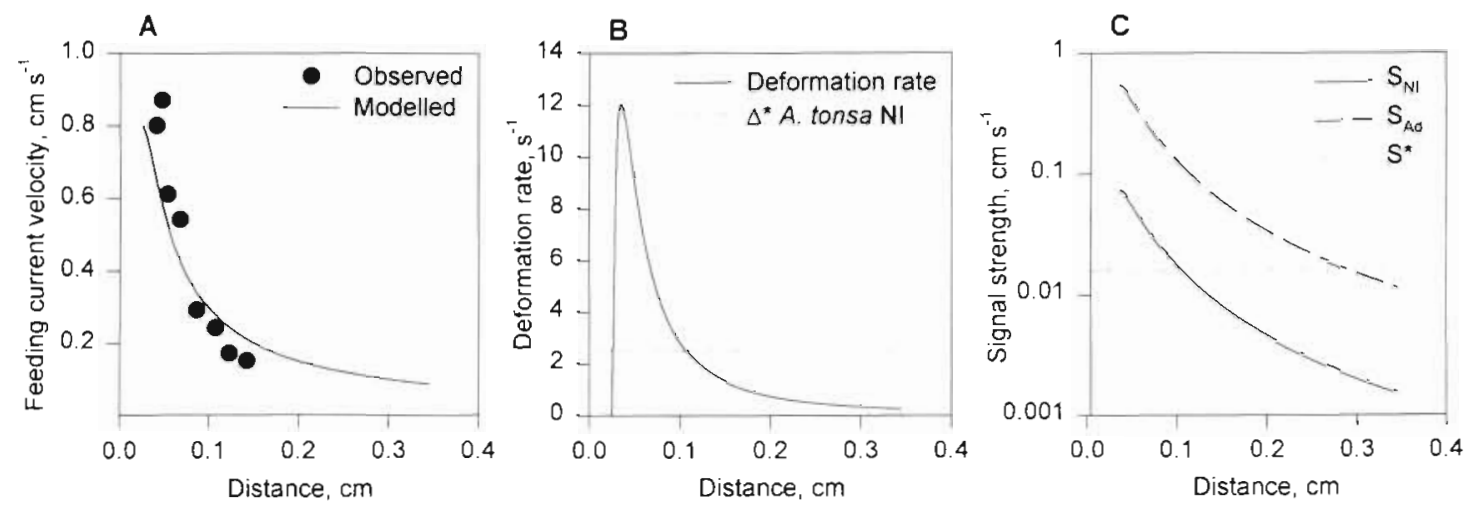

Fig. 9. (A) Modelled (line) and observed (dots) attenuation of feeding current velocity directly in front of Centropages typicus. Data from Tiselius \& Jonsson (1990) and model from Kiørboe \& Visser (1999). (B) Modelled attenuation of deformation rate directly in front of $C$. typicus. The horizontal line indicates the threshold deformation rate $\left(\Delta^{*}\right.$ ) for Acartia tonsa nauplius stage I. (C) Signal strength of $A$. tonsa nauplius stage I $\left(\mathrm{S}_{\mathrm{NI}}\right)$ and adult females $\left(\mathrm{S}_{\mathrm{Ad}}\right)$ in the feeding current of $C$. typicus as a function of distance. The horizontal line shows the threshold signal strength $\left(S^{*}\right)$ for $A$. tonsa

atively constant, $\sim 5$ to $15 \mathrm{~s}^{-1}$ (Table 6 ), thus allowing detection and subsequent escape of copepods with threshold deformation rates less that. Similar arguments suggest that a simple cruise strategy would allow copepods with threshold deformation rates less than 5 to $10 \mathrm{~s}^{-1}$ to detect their predator long before the predator is within striking distance (Kiørboe \& Visser 1999). It thus appears that realised threshold deformation rates fall within the relatively narrow window between turbulent deformation rates, and the rate required for efficient predator detection.

In the course of the evolutionary arms race, some planktonic predators have developed tactics to circumvent copepod predator detection capabilities. For example, the escape of a nauplius in a feeding current may produce an attack signal to the predatory copepod, as has been described in one species (Yen \& Strickler 1996). Alternatively, an ambush type predatory strategy, in which the predator sinks slowly through the water while searching for prey, may be adopted. Many copepods, including Centropages typicus (Tiselius \& Jonsson 1990) master both feeding current and ambush feeding strategies. The sinking velocities of $C$. typicus is about $0.1 \mathrm{~cm} \mathrm{~s}^{-1}$, which is less than the critical move velocity for predator detection in Acartia NI, $\sim 0.17 \mathrm{~cm}$ $\mathrm{s}^{-1}$ (calculated as $8 c \Delta * / 3$, where $c$ is predator radius, here assumed to be $0.025 \mathrm{~cm}$, Kiørboe \& Visser 1999). Thus, such a predatory copepod may almost bump into an Acartia nauplius without being detected. Similarly, many fish larval cruise predators, for example, decelerate upon prey detection and thus have sufficiently low final approach velocities to prevent being detected (Viitasalo et al. 1998, Kiorboe \& Visser 1999). Thus the ability of copepods to perceive fluid deformation rates and remotely detect predators reduces their risk of being eaten, but does not eliminate it.
Acknowledgements. We thank Elith Pedersen and Mogens Busse for help in designing and constructing our hydromechanical devices and David Fields and Per Jonsson for commenting on the manuscript. Financial support was received from The Danish Natural Science Research Council (\# 9502163) and from the EU-MAST project KEYCOP (\# MAS3CT97-0148).

\section{LITERATURE CITED}

Andrews JC (1983) Deformation of the active space in the low Reynolds number feeding current of calanoid copepods. Can J Fish Aquat Sci 40:1293-1302

Berggreen U, Hansen B, Kiørboe T (1988) Food size spectra, ingestion and growth of the copepod Acartia tonsa during development: implications for the determination of copepod production. Mar Biol 99:341-352

Costello JH, Strickler JR, Marrasé C, Trager G, Zeller R, Freise AJ (1990) Grazing in a turbulent environment: behavioural response of a calanoid copepod Centropages hamatus. Proc Natl Acad Sci USA 87:1648-1652

Fields DM, Yen J (1993) Outer limits and inner structure: the 3-dimensional flow field of Pleuromamma xiphias (Calanoida: Metridinidae). Bull Mar Sci 53:84-95

Fields DM. Yen J (1996) The escape behaviour of Pleuromamma xiphias in response to a quantifiable fluid mechanical disturbance. In: Lenz PH, Hartline DK, Purcell JE, Macmillan DL (eds) Zooplankton: sensory ecology and physiology, Vol 1. Gordon and Breach Publishers, Amsterdam, p 323-340

Fields DM, Yen J (1997a) The escape behaviour of marine copepods in response to a quantifiable fluid mechanical disturbance. J Plankton Res 19:1289-1304

Fields DM, Yen J (1997b) Implications of the feeding current structure of Euchaete rimana, a carnivorous pelagic copepod, on the spatial orientation of the prey. J Plankton Res 19:79-95

Haury LR, Kenyon DE, Brooks (1980) Experimental evaluation of the avoidance reaction of Calanus finmarchicus. J Plankton Res 2:187-202

Heuch PA, Karlsen HE (1997) Detection of infrasonic water oscillations by copepodids of Lepeoptherius salmonis (Copepoda: Caligida). J Plankton Res 19:735-747 
Jackson GA (1993) Particle trajectories in a rotating cylinder; Implications for aggregation incubations. Deep-Sea Res 41:429-437

Jonsson PR, André C, Lindegarth M (1991) Swimming behaviour of marine bivalve larvae in a flume boundary-layer flow: evidence for near-bottom confinement. Mar Ecol Prog Ser 79:67-76

Jonsson PR, Tiselius P (1990) Feeding behaviour, prey detection and capture efficiency of the copepod Acartia tonsa feeding on planktonic ciliates. Mar Ecol Prog Ser 60:35-44

Kerfoot WC (1978) Combat between predatory copepods and their prey: Cyclops, Epischura and Bosmina. Limnol Oceanogr 23:1089-1102

Kiørboe T, Saiz E (1995) Planktivorous feeding in calm and turbulent environments, with emphasis on copepods. Mar Ecol Prog Ser 122:135-145

Kiørboe T, Visser AW (1999) Predator and prey perception in copepods due to hydromechanical signals. Mar Ecol Prog Ser 179:81-95

Kirk KL, Gilbert JJ (1988) Escape behaviour of Polyathra in response to artificial flow stimuli. Bull Mar Sci 53:96-105

Landry MR (1978) Predatory feeding behaviour of a marine copepod, Labidocera trispinosa. Limnol Oceanogr. 23: $1103-1113$

Mackas DL, Sefton H, Miller CB, Raich A (1993) Vertical habitat partitioning by large calanoid copepods in the oceanic Subarctic Pacific during spring. Prog Oceanogr 32: 259-294

Mauchline M (1998) The biology of calanoid copepods. Adv Mar Biol 33:1-710

Maxey MR, Riley JJ (1983) Equation of motion for a small rigid sphere in a nonuniform flow. Phys Fluids 26: $883-889$

Paffenhöfer GA, Lewis KD (1990) Perceptive performance and feeding behaviour of calanoid copepods. J Plankton Res 12:933-946

Saiz E, Alcaraz M (1992) Free-swimming behaviour of Acartia clausi (Copepoda: Calanoida) under turbulent water motion. Mar Ecol Prog Ser 80:229-236

Editorial responsibility: Otto Kinne (Editor),

Oldendorf/Luhe, Germany
Schröder VR (1967) Verhalten von Cyclops abyssorum in der Strömung. Arch Hydrobiol Suppl 33:84-91

Singarajah KV (1969) Escape reactions of zooplankton: the avoidance of a pursuing siphon tube. J Exp Mar Biol Ecol $3: 171-178$

Singarajah KV (1975) Escape reactions of zooplankton: effects of light and turbulence. J Mar Biol Assoc UK 55:627-639

Strickler JR (1985) Feeding currents in calanoid copepods: two new hypotheses. In: Laverack MS (ed) Physiological adaptations of marine animals. Symp Soc Exp Biol 89: $459-485$

Strickler JR, Bal AK (1973) Setae of the first antennae of the copepod Cyclops scutifer (Sars): their structure and importance. Proc Nat Acad Sci USA 70:2656-2659

Støttrup JG, Richardson K, Kirkegaard E, Pihl NJ (1986) The cultivation of Acartia tonsa Dana for use as a live food source for marine fish larvae. Aquaculture 52:87-96

Tiselius P, Jonsson P (1990) Foraging behaviour of six calanoid copepods: observations and hydrodynamic analysis. Mar Ecol Prog Ser 66:23-33

Van Duuren FA (1968) Defined velocity gradient model flocculator. J Sanit Eng Div Proc A.m Soc Civ Eng 94 (SA4): 671-682

Vogel S (1994) Life in moving fluids, 2nd edn. Princeton University Press, Princeton

Viitasalo M, Kiørboe T, Flinkman J, Pedersen LW, Visser AW (1998) Predation vulnerability of planktonic copepods: consequences of predator foraging strategies and prey sensory abilities. Mar Ecol Prog Ser 175:129-142

Yen J, Fields DM (1992) Escape response of Acartia hudsonica (Copepoda) nauplii from flow field of Temora longicornis (Copepoda). Arch Hydrobiol Beih Ergeb Limnol 36: $123-134$

Yen J, Strickler JR (1996) Advertisement and concealment in the plankton: what makes a copepod hydrodynamically conspicuous? Invertebr Biol 115:191-205

Yen J, Lenz PH, Gassie DV, Hartline DK (1992) Mechanoreception in marine copepods: electrophysiological studies on the first antennae. J Plankton Res 14:459-512

Submitted: August 3, 1998; Accepted: November 18, 1998 Proofs received from author(s): March 25, 1999 